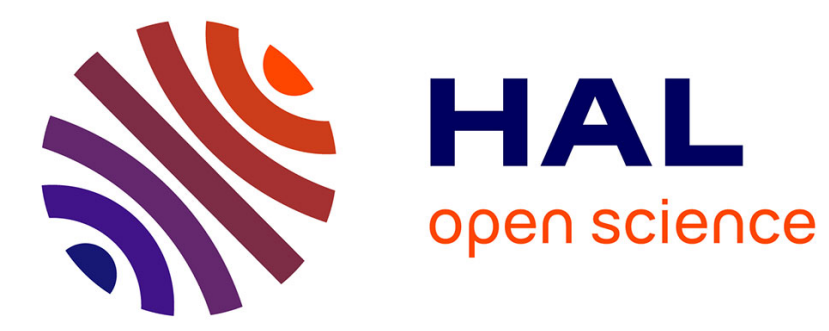

\title{
Logistic regression revisited: belief function analysis
}

Thierry Denoeux

\section{To cite this version:}

Thierry Denoeux. Logistic regression revisited: belief function analysis. 5th International Conference on Belief Functions (BELIEF 2018), Sep 2018, Compiègne, France. pp.57-64, 10.1007/978-3-31999383-6_8. hal-02553205

\section{HAL Id: hal-02553205 \\ https://hal.science/hal-02553205}

Submitted on 24 Apr 2020

HAL is a multi-disciplinary open access archive for the deposit and dissemination of scientific research documents, whether they are published or not. The documents may come from teaching and research institutions in France or abroad, or from public or private research centers.
L'archive ouverte pluridisciplinaire HAL, est destinée au dépôt et à la diffusion de documents scientifiques de niveau recherche, publiés ou non, émanant des établissements d'enseignement et de recherche français ou étrangers, des laboratoires publics ou privés. 


\title{
Logistic regression revisited: belief function analysis
}

\author{
Thierry Denoeux \\ Université de Technologie de Compiègne, CNRS, \\ UMR 7253 Heudiasyc, France \\ tdenoeux@utc.fr
}

\begin{abstract}
We show that the weighted sum and softmax operations performed in logistic regression classifiers can be interpreted in terms of evidence aggregation using Dempster's rule of combination. From that perspective, the output probabilities from such classifiers can be seen as normalized plausibilities, for some mass functions that can be laid bare. This finding suggests that the theory of belief functions is a more general framework for classifier construction than is usually considered.
\end{abstract}

Keywords: Evidence theory, Dempster-Shafer theory, classification, machine learning.

\section{Introduction}

In the last twenty years, the Dempster-Shafer (DS) theory of belief functions has been increasingly applied to classification. One direction of research is classifier fusion: classifier outputs are expressed as belief functions and combined by Dempster's rule or any other rule (see, e.g., [8], [1], [7]). Another approach is to design evidential classifiers, which can be defined as classifiers built from basic principles of DS theory. Typically, an evidential classifier has the structure depicted in Figure 1: when presented by a feature vector $x$, the system computes $k$ mass functions $m_{1}, \ldots, m_{k}$ defined on the set $\Theta$ of classes, based on a learning set. These mass functions are then combined using Dempster's rule, or any other rule. The first evidential classifier was the evidential $k$-nearest neighbor classifier [3], in which mass functions $m_{j}$ are constructed from the $k$ nearest neighbor of $x$, and combined by Dempster's rule. In the evidential neural network classifier [5], a similar principle is applied, but mass functions are constructed based on the distances to prototypes, and the whole system is trained to minimize an error function.

In this paper, we show that not only these particular distance-based classifiers, but also a broad class of widely-used classifiers, including logistic regression and its nonlinear extensions, can be seen as evidential classifiers. This finding leads us to the conclusion that DS theory is a much more general framework for classifier construction than was initially believed.

The rest of the paper is organized as follows. Some background definitions will first be recalled in Section 2. A general model of feature-based evidence will 


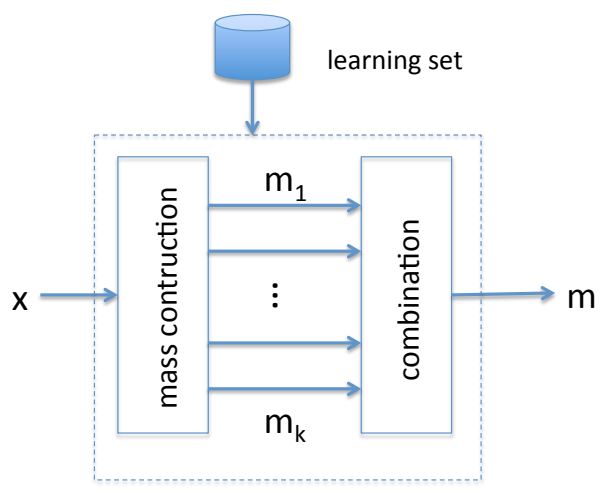

Fig. 1. Basic structure of an evidential classifier.

be described in Section 3, where we will show that the normalized plausibility function, after combining the evidence of $J$ features, is identical to the output of logistic regression. The recovery of the full mass function will then be addressed, and a simple example will be given in Section 4. Section 5 will conclude the paper.

\section{Background}

In this section, we first recall some basic notions and definitions needed in the rest of the paper. The notion of weight of evidence will first be recalled in Section 2.1, and some notations for logistic regression will be introduced in Section 2.2.

\subsection{Weights of evidence}

Let us consider a simple mass function $m$ on a frame $\Theta$, such that

$$
m(A)=s, \quad m(\Theta)=1-s,
$$

where $s$ is a degree of support in [0,1]. Typically, such a mass function represents some elementary piece of evidence supporting hypothesis $A$. Shafer [9, page 77] defines the weight of this evidence as $w=-\ln (1-s)$. Conversely, we thus have $s=1-\exp (-w)$. The rationale for this definition is that weights of evidence are additive: if $m_{1}$ and $m_{2}$ are two simple mass functions focussed on the same subset $A$, with weights $w_{1}$ and $w_{2}$, then the orthogonal sum $m_{1} \oplus m_{2}$ corresponds to the weight $w_{1}+w_{2}$. If we denote a simple mass function with focal set $A$ and weight $w$ by $A^{w}$, we thus have $A^{w_{1}} \oplus A^{w_{2}}=A^{w_{1}+w_{2}}$. It follows that any separable mass function can be written as $m=\bigoplus_{\emptyset \neq A \subset \Theta} A^{w_{A}}$, where $w_{A}$ is the weight of evidence pointing to $A$. We note that, in [6], following [10], we used the term "weight" for $-\ln w$. As we will see, the additivity property is central in our analysis: we thus stick to Shafer's terminology and notation in this paper. 


\subsection{Logistic regression}

Consider a multi-category ${ }^{1}$ classification problem with $J$-dimensional feature vector $x=\left(x_{1}, \ldots, x_{J}\right)$ and class variable $Y \in \Theta=\left\{\theta_{1}, \ldots, \theta_{K}\right\}$ with $K>2$. In the logistic regression model, we assume the logarithms of the posterior class probabilities $\left.\mathbb{P}\left(Y=\theta_{k}\right) \mid x\right)$ to be affine functions of $x$, i.e.,

$$
\ln \mathbb{P}\left(Y=\theta_{k} \mid x\right)=\sum_{j=1}^{J} \beta_{j k} x_{j}+\beta_{0 k}+\gamma, \quad \forall k \in \llbracket 1, K \rrbracket,
$$

where $\beta_{j k}, j=0, \ldots, J$ are parameters and $\gamma$ is a constant. Using the equation $\sum_{k=1}^{K} \mathbb{P}\left(Y=\theta_{k} \mid x\right)=1$, we easily get the following expressions for the posterior probabilities,

$$
\mathbb{P}\left(Y=\theta_{k} \mid x\right)=\frac{\exp \left(\sum_{j=1}^{J} \beta_{j k} x_{j}+\beta_{0 k}\right)}{\sum_{l=1}^{K} \exp \left(\sum_{j=1}^{J} \beta_{j l} x_{j}+\beta_{0 l}\right)} .
$$

This transformation from arbitrary real quantities (1) to probabilities is sometimes referred to as the softmax transformation. Parameters $\beta_{j k}$ are usually estimated by maximizing the conditional likelihood. In feedforward neural networks with a softmax output layer, a similar approach is used, with variables $x_{j}$ defined as the outputs of the last hidden layer of neurons. These variables are themselves defined as complex nonlinear functions of the input variables, which are optimized together with the decision layer weights $\beta_{j k}$. Logistic regression is functionally equivalent to a feedforward neural network with no hidden layer.

\section{Model}

We consider a multi-category classification problem as described in Section 2.2. We assume that each feature $x_{j}$ provides some evidence about the class variable $Y$. For each $\theta_{k}$, the evidence of feature $x_{j}$ points either to the singleton $\left\{\theta_{k}\right\}$ or to its complement $\overline{\left\{\theta_{k}\right\}}$, depending on the sign of

$$
w_{j k}=\beta_{j k} x_{j}+\alpha_{j k},
$$

where $\left(\beta_{j k}, \alpha_{j k}\right), k=1, \ldots, K, j=1, \ldots, J$ are parameters. The weights of evidence for $\left\{\theta_{k}\right\}$ and $\overline{\left\{\theta_{k}\right\}}$ are, respectively,

$$
w_{j k}^{+}=\left(w_{j k}\right)_{+} \quad \text { and } \quad w_{j k}^{-}=\left(w_{j k}\right)_{-},
$$

where $(\cdot)_{+}$and $(\cdot)_{-}$denote, respectively, the positive and the negative parts. For each feature $x_{j}$ and each class $\theta_{k}$, we thus have two simple mass functions

$$
m_{j k}^{+}=\left\{\theta_{k}\right\}^{w_{j k}^{+}} \quad \text { and } \quad m_{j k}^{-}=\overline{\left\{\theta_{k}\right\}} w_{j k}^{-}
$$

\footnotetext{
${ }^{1}$ The case of binary classification with $K=2$ classes requires a separate treatment.
} Due to space constaints, we focus on the multi-category case in this paper. 
Assuming these mass functions to be independent, they can be combined by Dempster's rule. Let

$$
m_{k}^{+}=\bigoplus_{j=1}^{J} m_{j k}^{+}=\left\{\theta_{k}\right\}^{w_{k}^{+}} \text {and } m_{k}^{-}=\bigoplus_{j=1}^{J} m_{j k}^{-}=\overline{\left\{\theta_{k}\right\}} w_{k}^{-}
$$

where

$$
w_{k}^{+}=\sum_{j=1}^{J} w_{j k}^{+} \quad \text { and } \quad w_{k}^{-}=\sum_{j=1}^{J} w_{j k}^{-} .
$$

The contour functions $p l_{k}^{+}$and $p l_{k}^{-}$associated, respectively, with $m_{k}^{+}$and $m_{k}^{-}$ are

$$
p l_{k}^{+}(\theta)= \begin{cases}1 & \text { if } \theta=\theta_{k}, \\ \exp \left(-w_{k}^{+}\right) & \text {otherwise }\end{cases}
$$

and

$$
p l_{k}^{-}(\theta)= \begin{cases}\exp \left(-w_{k}^{-}\right) & \text {if } \theta=\theta_{k} \\ 1 & \text { otherwise }\end{cases}
$$

Now, let

$$
m^{+}=\bigoplus_{k=1}^{K} m_{k}^{+} \quad \text { and } \quad m^{-}=\bigoplus_{k=1}^{K} m_{k}^{-},
$$

and let $p l^{+}$and $p l^{-}$be the corresponding contour functions. We have

$$
\begin{aligned}
p l^{+}\left(\theta_{k}\right) & \propto \prod_{l=1}^{K} p l_{l}^{+}\left(\theta_{k}\right)=\exp \left(-\sum_{l \neq k} w_{l}^{+}\right)=\exp \left(-\sum_{l=1}^{K} w_{l}^{+}\right) \exp \left(w_{k}^{+}\right) \\
& \propto \exp \left(w_{k}^{+}\right),
\end{aligned}
$$

and

$$
p l^{-}\left(\theta_{k}\right) \propto \prod_{l=1}^{K} p l_{l}^{-}\left(\theta_{k}\right)=\exp \left(-w_{k}^{-}\right)
$$

Finally, let $m=m^{+} \oplus m^{-}$and let $p l$ be the corresponding contour function. We have

$$
\begin{aligned}
p l\left(\theta_{k}\right) & \propto p l^{+}\left(\theta_{k}\right) p l^{-}\left(\theta_{k}\right) \propto \exp \left(w_{k}^{+}-w_{k}^{-}\right) \\
\propto \exp \left(\sum_{j=1}^{J} w_{j k}\right) & =\exp \left(\sum_{j=1}^{J} \beta_{j k} x_{j}+\sum_{j=1}^{J} \alpha_{j k}\right) .
\end{aligned}
$$

Let $p$ be the probability mass function induced from $m$ by the plausibilityprobability transformation [2], and let

$$
\beta_{0 k}=\sum_{j=1}^{J} \alpha_{j k}
$$


We have

$$
p\left(\theta_{k}\right)=\frac{\exp \left(\sum_{j=1}^{J} \beta_{j k} x_{j}+\beta_{0 k}\right)}{\sum_{l=1}^{K} \exp \left(\sum_{j=1}^{J} \beta_{j l} x_{j}+\beta_{0 l}\right)},
$$

which is equivalent to (2). We thus have proved that the output probabilities computed by a logistic regression classifier can be seen as the normalized plausibilities obtained after combining elementary mass functions (5) by Dempster's rule: these classifiers are, thus, evidential classifiers as defined in Section 1.

\section{Recovering the mass function}

Having shown that the output probabilities of logistic regression classifiers are normalized plausibilities, it is interesting to recover the underlying output mass function, defined as

$$
m=\bigoplus_{k=1}^{K}\left(\left\{\theta_{k}\right\}^{w_{k}^{+}} \oplus \overline{\left\{\theta_{k}\right\}} w^{-}\right) .
$$

Its complete expression can be derived (after some tedious calculation), but it cannot be given here for lack of space.

There is, however, a difficulty related to the identifiability of the weights $w_{k}^{+}$ and $w_{k}^{-}$. First, parameters $\beta_{j k}$ are not themselves identifiable, because adding any constant vector $\boldsymbol{c}$ to each vector $\beta_{k}=\left(\beta_{0 k}, \ldots, \beta_{J k}\right)$ produces the same normalized plausibilities (8). Secondly, for given $\beta_{0 k}$, any $\alpha_{j k}$ verifying (7) will yield the same probabilities (8). This problem is addressed in the next section.

\subsection{Identification}

To identifying the underlying output mass function, we propose to apply the Least Commitment Principle, by searching for the mass function $m^{*}$ of the form (9) verifying (8) and such that the sum of the squared weights of evidence is minimum. More precisely, let $\left\{\left(x_{i}, y_{i}\right)\right\}_{i=1}^{n}$ be a learning set, let $\widehat{\beta}_{j k}$ be the maximum likelihood estimates of the weights $\beta_{j k}$, and let $\boldsymbol{\alpha}$ denote the vector of parameters $\alpha_{j k}$. Any $\beta_{j k}^{*}=\widehat{\beta}_{j k}+c_{j}$ will verify (8). The parameter values $\beta_{j k}^{*}$ and $\alpha_{j k}^{*}$ minimizing the sum of the squared weights of evidence can thus be found by solving the following minimization problem

$$
\min f(\boldsymbol{c}, \boldsymbol{\alpha})=\sum_{i=1}^{n} \sum_{j=1}^{J} \sum_{k=1}^{K}\left[\left(\widehat{\beta}_{j k}+c_{j}\right) x_{i j}+\alpha_{j k}\right]^{2}
$$

subject to

$$
\sum_{j=1}^{J} \alpha_{j k}=\widehat{\beta}_{0 k}+c_{0}, \quad \forall k \in \llbracket 1, K \rrbracket .
$$


In $(10), x_{i j}$ denotes the value of feature $j$ for learning vector $x_{i}$. Developing the square in (10), we get

$$
f(\boldsymbol{c}, \boldsymbol{\alpha})=\sum_{j, k}\left(\widehat{\beta}_{j k}+c_{j}\right)^{2}\left(\sum_{i} x_{i j}^{2}\right)+n \sum_{j, k} \alpha_{j k}^{2}+2 \sum_{j, k}\left(\widehat{\beta}_{j k}+c_{j}\right) \alpha_{j k} \sum_{i} x_{i j} .
$$

Assuming that the input variables $x_{j}$ have been centered, we have $\sum_{i} x_{i j}=0$ and $\sum_{i} x_{i j}^{2}=s_{j}^{2}$, where $s_{j}^{2}$ is the empirical variance of feature $x_{j}$. Eq. (12) then simplifies to

$$
f(\boldsymbol{c}, \boldsymbol{\alpha})=\sum_{j, k} s_{j}^{2}\left(\widehat{\beta}_{j k}+c_{j}\right)^{2}+n \sum_{j, k} \alpha_{j k}^{2}
$$

Due to constraint (11), for any $c_{0}$, the second term in the right-hand side of (13) is minimized for $\alpha_{j k}=\frac{1}{J}\left(\widehat{\beta}_{0 k}+c_{0}\right)$, for all $j \in \llbracket 1, J \rrbracket$ and $k \in \llbracket 1, K \rrbracket$. Hence, the problem becomes

$$
\min _{\boldsymbol{c}} f(\boldsymbol{c})=\sum_{j=1}^{J} s_{j}^{2}\left\{\sum_{k=1}^{K}\left(\widehat{\beta}_{j k}+c_{j}\right)^{2}\right\}+\frac{n}{J} \sum_{k=1}^{K}\left(\widehat{\beta}_{0 k}+c_{0}\right)^{2} .
$$

Each of the $J+1$ terms in this sum can be minimized separately. The solution can easily be found to be

$$
c_{j}^{*}=-\frac{1}{K} \sum_{k=1}^{K} \widehat{\beta}_{j k}, \quad \forall j \in \llbracket 0, J \rrbracket
$$

The optimum coefficients are, thus,

$$
\beta_{j k}^{*}=\widehat{\beta}_{j k}-\frac{1}{K} \sum_{l=1}^{K} \widehat{\beta}_{j l}, \quad \forall j \in \llbracket 0, J \rrbracket, \forall k \in \llbracket 1, K \rrbracket
$$

and

$$
\alpha_{j k}^{*}=\beta_{0 k}^{*} / J, \quad \forall j \in \llbracket 1, J \rrbracket, \forall k \in \llbracket 1, K \rrbracket .
$$

To get the least committed mass function $m^{*}$ with minimum sum of squared weights of evidence and verifying (8), we thus need to center the rows of the $(J+1) \times K$ matrix $B=\left(\beta_{j k}\right)$, set $\alpha_{j k}^{*}$ according to $(14)$, and compute the weights of evidence $w_{k}^{-}$and $w_{k}^{+}$from $(3),(4)$ and (6).

\subsection{Example}

As a simple example, let us consider simulated data with $J=1$ feature, $K=3$ classes, and Gaussian conditional distributions $X \mid \theta_{k} \sim \mathcal{N}\left(\mu_{k}, 1\right)$, with $\mu_{1}=-1$, $\mu_{2}=0$ and $\mu_{3}=1$. We randomly generated 10,000 from each of the three conditional distributions, we standardized the data and we trained a logistic regression classifier on these data. Decisions are usually based on the posterior 
class probabilities $\mathbb{P}\left(\theta_{k} \mid x\right)$ displayed in Figure 2(a). Figure 3 shows the underlying masses, computed as explained in Section 4.1. As we can see, masses are assigned to subsets of classes in regions where these classes overlap, as could be expected. Figure 2(b) shows the contour functions $p l\left(\theta_{k} \mid x\right)$ vs $x$. Interestingly, the graphs of these functions have quite different shapes, as compared to those of the posterior probabilities shown in Figure 2(a). Whereas decisions with probabilistic classifiers are classically based on minimum expected loss, seeing logistic regression classifiers as evidential classifiers opens the possibility to experiment with other rules such as minimum lower or upper expected loss [4] or interval dominance [11].

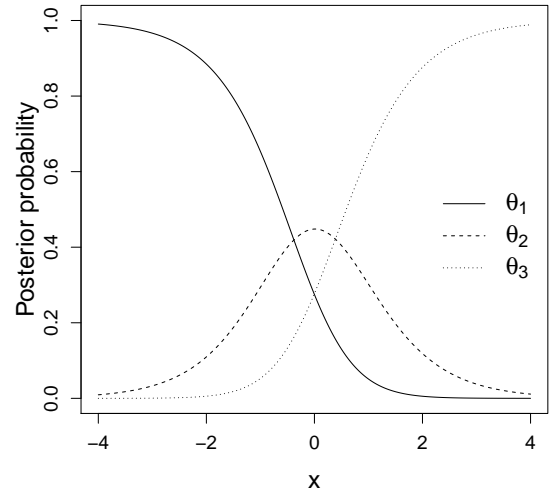

(a)

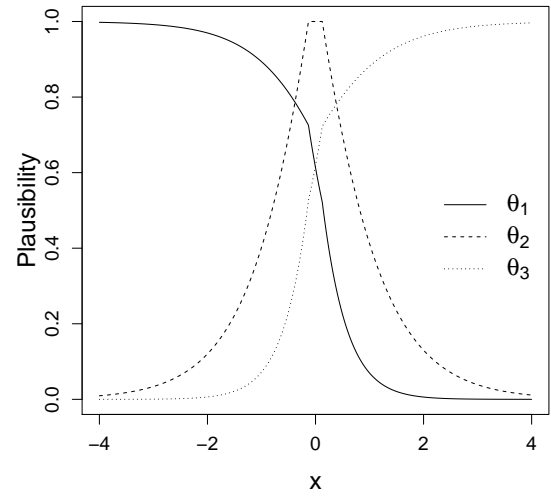

(b)

Fig. 2. Posterior class probabilities $\mathbb{P}\left(\theta_{k} \mid x\right)$ (a) and contour functions $p l\left(\theta_{k} \mid x\right)$ for the logistic regression example.

\section{Conclusions}

We have shown that logistic regression classifiers and also, as a consequence, generalized linear classifiers such as feedforward neural network classifiers, which essentially perform logistic regression in the output layer, can be seen as pooling evidence using Dempster's rule of combination. This finding may have important implications, as it opens the way to a DS analysis of many widely used classifiers, beyond the particular distance-based classifiers introduced in [3] and [5]. In future work, we will deepen this analysis by exploring the consequences of viewing neural network classifiers as evidential classifiers, in terms of decision strategies, classifier fusion, and handling missing or uncertain inputs, among other research directions. 


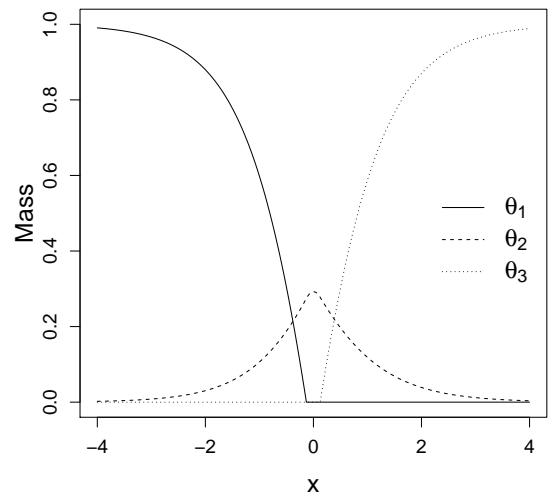

(a)

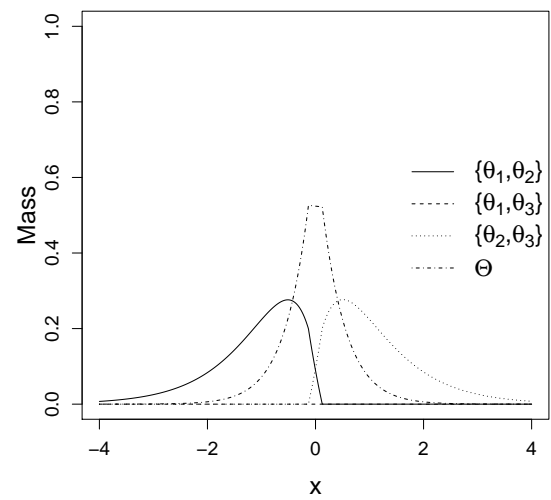

(b)

Fig. 3. Masses on singletons (a) and compound hypotheses (b) vs. $x$ for the logistic regression example.

\section{References}

1. Y. Bi, J. Guan, and D. Bell. The combination of multiple classifiers using an evidential reasoning approach. Artificial Intelligence, 172(15):1731-1751, 2008.

2. B. R. Cobb and P. P. Shenoy. On the plausibility transformation method for translating belief function models to probability models. International Journal of Approximate Reasoning, 41(3):314-330, 2006.

3. T. Denœux. A $k$-nearest neighbor classification rule based on Dempster-Shafer theory. IEEE Trans. on Systems, Man and Cybernetics, 25(05):804-813, 1995.

4. T. Denœux. Analysis of evidence-theoretic decision rules for pattern classification. Pattern Recognition, 30(7):1095-1107, 1997.

5. T. Denœux. A neural network classifier based on Dempster-Shafer theory. IEEE Trans. on Systems, Man and Cybernetics A, 30(2):131-150, 2000.

6. T. Denœux. Conjunctive and disjunctive combination of belief functions induced by non distinct bodies of evidence. Artificial Intelligence, 172:234-264, 2008.

7. B. Quost, M.-H. Masson, and T. Denœux. Classifier fusion in the Dempster-Shafer framework using optimized t-norm based combination rules. International Journal of Approximate Reasoning, 52(3):353-374, 2011.

8. G. Rogova. Combining the results of several neural network classifiers. Neural Networks, 7(5):777-781, 1994.

9. G. Shafer. A mathematical theory of evidence. Princeton University Press, Princeton, N.J., 1976.

10. P. Smets. The canonical decomposition of a weighted belief. In Int. Joint Conf. on Artificial Intelligence, pages 1896-1901, San Mateo, Ca, 1995. Morgan Kaufman.

11. M. C. Troffaes. Decision making under uncertainty using imprecise probabilities. International Journal of Approximate Reasoning, 45(1):17 - 29, 2007. 